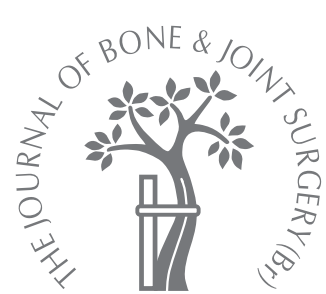

B. Christen, P. V. Heesterbeek, A. Wymenga, U. Wehrli

From Spital BernZiegler, Bern, Switzerland

\title{
Posterior cruciate ligament balancing in total knee replacement
}

\author{
THE QUANTITATIVE RELATIONSHIP BETWEEN TIGHTNESS OF \\ THE FLEXION GAP AND TIBIAL TRANSLATION
}

\footnotetext{
a. Christen, MD, MHA, Orthopaedic Surgeon Orthopaedic Clinic Salemspital, Schänzlistrasse 39, CH-3000 Berne 25, Switzerland.

In P. V. Heesterbeek, MSc, Scientific Researcher Department of Research, Development and Education A. Wymenga, MD, PhD, Orthopaedic Surgeon Department of Orthopaedics Sint Maartenskliniek, Postbox 9011, 6500 GM Nijmegen, The Netherlands.
}

- U. Wehrli, MD, Orthopaedic Surgeon (Retired)

Department of Orthopaedics Spital Bern Ziegler, Route de la Crausa 35, 1789 Lugnorre,

Switzerland.

Correspondence should be sent to Ms. P. V. Heesterbeek; e-mail: p.heesterbeek@maartensklinie k.nl

()2007 British Editorial Society of Bone and Joint Surgery doi:10.1302/0301-620X.89B8. $18976 \$ 2.00$

$J$ Bone Joint Surg $[B r]$ 2007;89-B:1046-50. Received 29 November 2006; Accepted after revision 9 May 2007

\begin{abstract}
We have examined the relationship between the size of the flexion gap and the anterior translation of the tibia in flexion during implantation of a posterior cruciate ligament (PCL)retaining BalanSys total knee replacement (TKR). In 91 knees, the flexion gap and anterior tibial translation were measured intra-operatively using a custom-made, flexible tensorspacer device.

The results showed that for each increase of $1 \mathrm{~mm}$ in the flexion gap in the tensed knee a mean anterior tibial translation of $1.25 \mathrm{~mm}$ (SD $0.79,95 \%$ confidence interval 1.13 to 1.37 ) was produced.

When implanting a PCL-retaining TKR the surgeon should be aware that the tibiofemoral contact point is related to the choice of thickness of the polyethylene insert. An additional thickness of polyethylene insert of $2 \mathrm{~mm}$ results in an approximate increase in tibial anterior translation of $\mathbf{2 . 5} \mathbf{~ m m}$ while the flexed knee is distracted with a force of between $100 \mathrm{~N}$ and $200 \mathrm{~N}$.
\end{abstract}

While there is no consensus on whether it is necessary to retain the posterior cruciate ligament (PCL), ${ }^{1}$ its retention in total knee replacement (TKR) gives excellent long-term results. ${ }^{2,3}$ In the knee, the PCL is the main restraint against posterior translation of the tibia, ${ }^{4}$ particularly so at about $90^{\circ}$ of flexion and with little effect on knee kinematics in either extension or high flexion. ${ }^{5,6}$ Most fibres of the PCL are under tension only at around $90^{\circ}$ of flexion ${ }^{4,7}$ and it controls the contact point of the medial femoral condyle on the tibia between approximately $60^{\circ}$ and $120^{\circ}$ of flexion. Recent MRI studies of the normal knee have shown that during a wide range of movement the medial femoral condyle behaves as a fixed circle with no anteroposterior (AP) translation on the tibia. ${ }^{8}$ In other words, there is no roll-back from between $10^{\circ}$ and $120^{\circ}$ of flexion. ${ }^{8}$ The contact point of the medial femoral condyle is maintained between $2 \mathrm{~mm}$ and $5 \mathrm{~mm}$ posterior to the middle of the medial tibial plateau, representing $54 \%$ to $60 \%$ of the AP diameter of the medial tibia. 8,9 The medial compartment of the knee is thus comparatively constrained, and this contact point should be restored during TKR in order to obtain normal kinematics. We suggest that after a PCL-retaining TKR, this position for the contact point is only achieved when the PCL is placed under adequate tension. In addition, the tension in the collateral ligaments also needs to be correct, both in flexion and extension, in order to achieve normal kinematics.

Several studies have shown the importance but also the difficulty of balancing the PCL in a PCL-retaining TKR. ${ }^{10-14}$ If the PCL has not been adequately balanced, complications may occur. A loose PCL may result in instability and pain. ${ }^{15-17}$ Alternatively, excessive tension in the PCL may restrict flexion or lead to high stress on polyethylene wear ${ }^{17}$ or even posteromedial subluxation. ${ }^{18}$ Incorrect tensioning has been associated with early catastrophic failure. ${ }^{19}$ However, a radiological study has shown that it is possible to balance a PCLretaining TKR to achieve a contact point at approximately $55 \%$ of the AP tibial plateau distance, ${ }^{20}$ thus achieving a contact point similar to that of the normal knee. ${ }^{8}$

Using a ligament-guided knee system with a ligament tensor which can apply a measured amount of tension, we have observed a considerable effect of the tensor force on the flexion gap. When the flexion gap was distracted with the tensor, the tibia moved anteriorly and the size of the gap increased. We could find no data in the literature which described this phenomenon quantitatively. Therefore, in order to investigate the dynamics of the flexion gap we developed a custom-made, flexible tensorspacer device which could measure the size of 


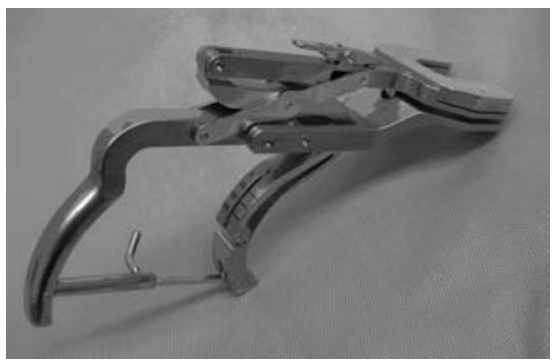

Fig. 1a

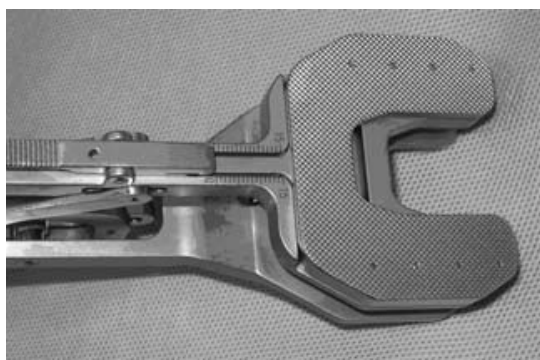

Fig. 1d

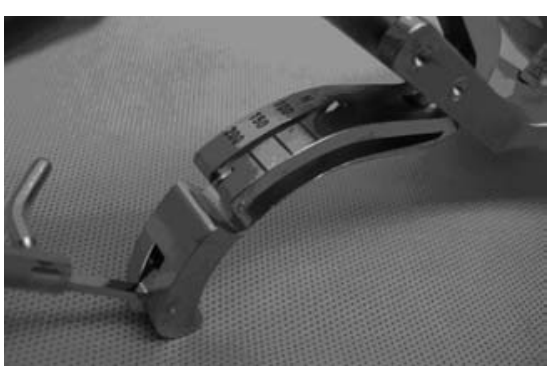

Fig. 1b

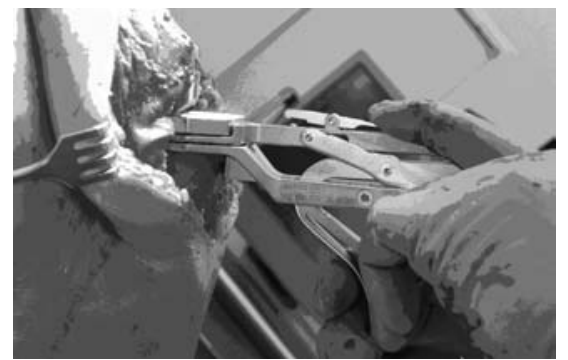

Fig. 1e

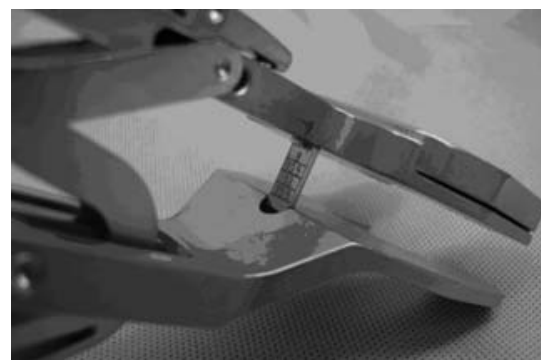

Fig. 1c

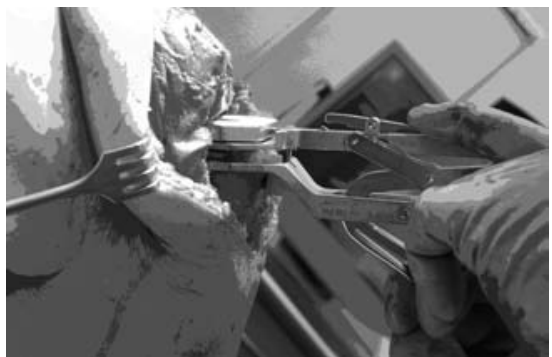

Fig. $1 f$

Photographs showing a) the posterior cruciate ligament tensioner, b) the handle which shows the amount of force used, c) measurement of the gap height, d) measurement of anterior translation, e) the tensioner in situ not tensioned, and f) the tensioner in situ, while tensioned (see text).

the flexion gap, distraction force and anterior translation of the tibia. Our aim was to describe the relation between the size and the anterior translation of the flexion gap during the implantation of a PCL-retaining total knee prosthesis.

\section{Patients and Methods}

In this prospective study we included 91 knees (59 right, 32 left) in 83 patients with a mean age of 71 years (SD 9.1) who were undergoing primary TKR. There were 23 men and 60 women with an intact PCL, as assessed by macroscopic inspection by the surgeon (BC, AW). Pre-operatively, 58 knees $(63.7 \%)$ had a varus deformity and $33(36.3 \%)$ a valgus deformity. A total condylar PCL-retaining ligamentguided BalanSys TKR (Mathys, Bettlach, Switzerland) was implanted in all the knees.

Operative technique. The tibial osteotomy was performed first with a posterior tibial slope of $7^{\circ}$. The insertion of the PCL was preserved by a bony island through a so-called 'V-cut'. After the tibial osteotomy, the necessary releases of the medial or lateral ligaments were made in extension with a double-spring tensioner in the knee which exerted a force of between $150 \mathrm{~N}$ and $200 \mathrm{~N}$. The knee was then flexed and the double tensioner was re-inserted and a force of $100 \mathrm{~N}$ to $150 \mathrm{~N}$ was applied. These tension values for extension and flexion were chosen based on clinical experience in the absence of any published guidance. The rotational position of the femur was determined by the tension of the collateral ligaments and a rectangular flexion gap was created by performing the posterior femoral osteotomies. After dorsal and central osteophytes had been removed, the experimental measurements were made.

Measurements. These were performed using a custommade BalanSys PCL Tensioner (Mathys) with an adjustable spacer block (Fig. 1). The minimum thickness of the tensor was $17 \mathrm{~mm}, 9 \mathrm{~mm}$ simulating the thickness of the femoral component, $2 \mathrm{~mm}$ the thickness of the tibial tray and $6 \mathrm{~mm}$ the minimum indication of the requirement for the polyethylene insert. The device showed the size of the flexion gap starting at $6 \mathrm{~mm}$ which is $2 \mathrm{~mm}$ less than the thinnest available polyethylene insert in the BalanSys system. The tensor was inserted with the knee at $90^{\circ}$ of flexion and $100 \mathrm{~N}, 150 \mathrm{~N}$ and $200 \mathrm{~N}$ of force were applied sequentially as indicated on the scale of the instrument. When the PCL tensioner was opened, the size of the PCL spacer increased and the requirement of the polyethylene thickness in millimetres was recorded. On the femoral side, a sliding plateau gave the amount of anterior tibial translation when the spacer was gradually opened. Since the direction of the fibres in the PCL is oblique, running from the inferoposterior tibia to the superoanterior medial femoral condyle, opening of the joint space with the tensor caused the tibia to move forwards as the PCL was tensioned (Fig. 2).

Using the scales on the spacer, the size of the flexion gap and the translation of the tibia were measured in millimetres for three distraction forces, $100 \mathrm{~N}, 150 \mathrm{~N}$ and $200 \mathrm{~N}$. The surgeon (BC, AW) progressively increased the tension 

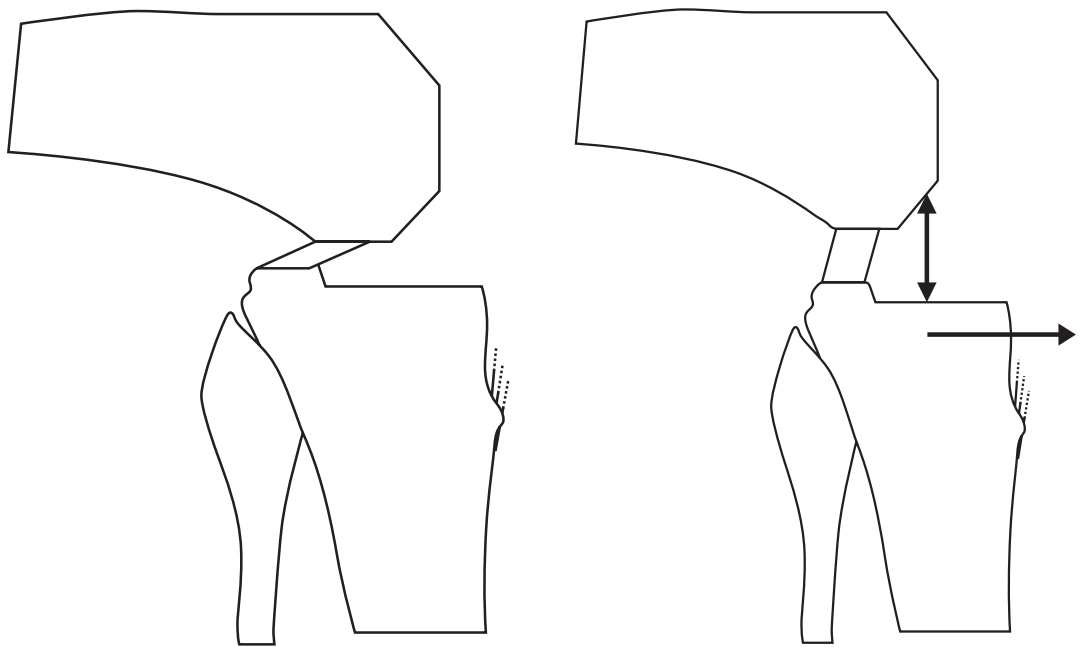

Fig. 2

Diagrams showing the orientation of the posterior cruciate ligament $(\mathrm{PCL})$ and relative movements of the femur in relation to the tibia. As the flexion gap increases by applied tension, the increase of tension in the PCL, results in pivoting of the tibia around the femoral insertion of the $\mathrm{PCL}$, leading to anterior translation of the tibia. between consecutive measurements within a single knee without returning to zero between measurements, i.e., measurements were made in a stair-wise fashion.

In order to identify the relationship between the size of the gap and anterior tibial translation, the progressive differences were calculated between $100 \mathrm{~N}$ and $150 \mathrm{~N}$ and between $150 \mathrm{~N}$ and $200 \mathrm{~N}$ in each individual knee. Deltagap1 and deltagap2 were the differences between the flexion gap at $100 \mathrm{~N}$ and $150 \mathrm{~N}$, and $150 \mathrm{~N}$ and $200 \mathrm{~N}$, respectively. The anterior tibial translations, deltatrans 1 and deltatrans2, were calculated in a similar manner. In order to calculate the amount of anterior tibial translation when the size of the gap increased by $1 \mathrm{~mm}$, the mathematical slopes (PCLslope1 and PCLslope2) were determined (deltatrans divided by deltagap).

Statistical analysis. The difference between PCLslope1 and PCLslope 2 was tested using a paired Student's $t$-test. If the deltagap was 0 , then the mathematical slope could not be calculated. Statistical analyses were performed using SPSS software (SPSS Inc., Chicago, Illinois) and the results were presented as the mean and SD, and the 95\% confidence intervals (CI) were calculated. The level of statistical significance was set at $\mathrm{p} \leq 0.05$ (Student's $t$-test).

\section{Results}

Measurements were obtained from all 91 knees at $100 \mathrm{~N}$ and $150 \mathrm{~N}$. Because of a weak PCL, one knee was not measured at $200 \mathrm{~N}$. Deltagap1 was 0 in five knees and deltagap2 was 0 in six knees, therefore it was not possible to calculate a mathematical slope for these knees.

The mean PCLslope1 ( $\mathrm{n}=86)$ was 1.17 (SD 0.80, 95\% CI 0.99 to 1.34$)$ and the mean PCLslope2 ( $\mathrm{n}=85$ ) was 1.33 (SD $0.77,95 \%$ CI 1.17 to 1.50 ). There was no statistical difference (Student's $t$-test, $\mathrm{p}=0.214$ ) between PCLslope1 and PCLslope2, and therefore the values for the mathematical slopes were analysed together, producing a mean PCLslope $(\mathrm{n}=171)$ of 1.25 (SD 0.79, 95\% CI
1.13 to 1.37$)$. This meant that for each increase of $1 \mathrm{~mm}$ in the flexion gap, a mean increase of $1.25 \mathrm{~mm}$ in anterior tibial translation occurred (Fig. 3). As the deltagap increased, the CI also widened.

\section{Discussion}

In this study there was a positive relationship between the increase in the size of the flexion gap and the anterior translation of the tibia, confirming our observations during surgery. The anterior tibial translation of $1.25 \mathrm{~mm}$ for each increase of $1 \mathrm{~mm}$ in the flexion gap can be explained by the oblique orientation of the PCL fibres. When the gap between the femur and the tibia was increased, the increase in tension in the PCL resulted in the tibia pivoting around the femoral insertion of the PCL thus leading to an anterior translation of the tibia relative to the femur.

The absence of a statistically significant difference between the mean PCLslope1 and PCLslope2 suggests that the relationship between anterior tibial translation and the size of the flexion gap was not dependent on the amount of force applied. We had expected more anterior tibial translation between $100 \mathrm{~N}$ and $150 \mathrm{~N}$ compared with $150 \mathrm{~N}$ and $200 \mathrm{~N}$, believing that the PCL fibres would become more vertical with higher forces resulting in less anterior tibial translation. However, this did not occur, possibly because the amount of force which was applied was not great enough to cause the PCL fibres to run in a purely vertical orientation. Based on clinical experience we limited the tensor force to $200 \mathrm{~N}$ to prevent damage to the ligaments.

We found some variation between individual patients which may have been caused by the different amount of resected tibial thickness for each knee. The size of the flexion gap was partly dependent on the initial tibial cut since it influenced the pretension of the PCL when the tensioner was inserted. For example, a conservative tibial cut led to a 


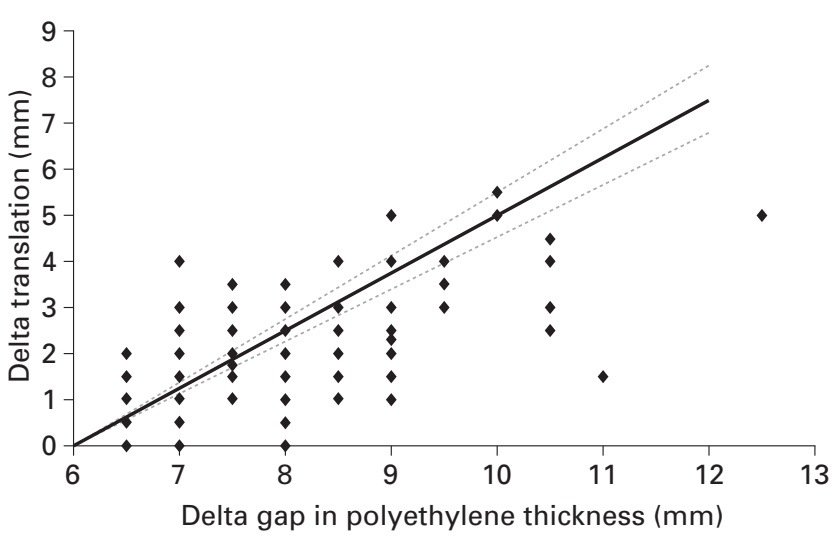

Fig. 3

Scatterplot of deltagap against deltatranslation $(n=171)$. The deltagap in polyethylene thickness is $6 \mathrm{~mm}$ plus the recorded measurement in gap size. The black line represents the mean PCLslope (1.25), and the dotted lines the upper and lower limits of the $95 \%$ confidence interval.

greater pretensioned PCL when the $11+6 \mathrm{~mm}$ tensioner was inserted, whereas a larger tibial cut left more space for the tensioner, with the result that the PCL was only tight after a greater amount of tension had been applied. This phenomenon might also have accounted for the fact that PCLslope1 and PCLslope 2 were 0 in a few knees. Similarly, at $100 \mathrm{~N}$ or $150 \mathrm{~N}$, the force might have been too small to induce an increase in the flexion gap if the initial tibial cut had been generous. With a conservative tibial cut, the flexion gap might have been at the maximum at $150 \mathrm{~N}$ and thus, any further increase in force would not have resulted in a further increase of the flexion gap as was seen when the PCLslope2 was 0 .

In addition to the variable thickness of the tibial resection, variation in the PCL morphotype may also have affected the results with the PCL fibres being more vertically orientated in some patients and having a more horizontal course in others. Furthermore, in very tall or obese patients, the increased weight of the limb would render the flexion gap harder to open at $100 \mathrm{~N}$ compared with a less heavy limb.

The measurements in our study were performed with an intact PCL. In a recent five-year follow-up study of 50 patients with a PCL-retaining BalanSys TKR (Mathys), no cases of posterior laxity were suggesting that the PCL remained functional. ${ }^{21}$ Others have questioned whether the PCL in an arthritic knee can be considered to be intact, ${ }^{22}$ and some authors found increased AP laxity at later followup. ${ }^{12}$ We believe that the use of a tensor is important for correct balancing of the flexion gap in PCL-retaining TKR. Despite the physical differences between individual knees, it was possible to investigate the relationship of the flexion gap to the translational movement of the contact point by normalising the data, that is dividing the change in translation by the change in flexion gap.
The problem of correct balancing of the PCL has been previously studied. Balancing of the PCL is essential to achieve good flexion and optimal contact point in the PCLretaining prosthetic knee. If the PCL is not adequately balanced, paradoxical roll-forward can occur. ${ }^{23}$ If a flexion gap is created without sufficient PCL tension, the femur will automatically slide forward. At the other extreme, if the PCL is too tight, the contact position will be excessively posterior. Changes in contact point also affect the range of knee flexion. ${ }^{24}$ For each additional millimetre of posterior femoral translation, an additional $1.4^{\circ}$ of flexion has been described but if the contact point is too posterior it will cause excessive tightening of the PCL and provoke pain and restrict flexion. ${ }^{23}$

Our findings give an explanation for the difficulty in achieving correct tension in the PCL and a correct contact point. Laboratory experiments have provided contradictory results in achieving a correctly functioning PCL. ${ }^{10,25-27}$ Also in clinical series with fixed-bearing inserts, several authors have reported failure to obtain correct balancing in all knees $^{11-13,28}$ and in AP-meniscal-bearing knee implants it has been shown to be even more crucial to obtain a correct balance. ${ }^{16,29,30}$ It should be appreciated that implants with flat fixed inserts or AP-meniscal inserts rely more on soft tissue while conforming dished inserts are more self-stabilising. ${ }^{31}$

Furthermore, fluoroscopic studies have shown frequent paradoxical anterior gliding in PCL-retaining knees, ${ }^{24,32}$ but the results seem to vary between surgeons. ${ }^{23}$ By contrast, two contemporary reports have shown the ability to create a correct contact point after PCL-retaining TKR. ${ }^{33,34}$ However, the method of balancing the PCL was not clearly reported. We feel that the presence of paradoxical movement is the consequence of an inadequately balanced PCL and collateral ligaments.

Our study illustrates the dynamic character of the flexion gap, but does not solve the balancing problem. We could not identify an ideal force for a tensioner from our data, but our clinical impression was that a force around $100 \mathrm{~N}$ was sufficient to achieve satisfactory AP stability intra-operatively and yielded a good post-operative range of flexion.

In conclusion, when implanting a PCL-retaining TKR the surgeon needs to be aware that the position of the tibiofemoral contact point is related to the choice of the thickness of the polyethylene insert. We found that for each increase of $1 \mathrm{~mm}$ in the flexion gap there was a corresponding mean increase in tibial translation of $1.25 \mathrm{~mm}$.

We wish to thank P. G. Anderson for her help and advice in the analysis and writing process, and also B. E. Groen for her suggestion in the data analysis. No benefits in any form have been received or will be received from a commercial party related directly or indirectly to the subject of this article.

\section{References}

1. Jacobs WC, Clement DJ, Wymenga AB. Retention versus removal of the posterior cruciate ligament in total knee replacement: a systematic literature review within the Cochrane framework. Acta Orthop 2005;76:757-68. 
2. Rand JA, Trousdale RT, IIstrup DM, Harmsen WS. Factors affecting the durability of primary total knee prostheses. J Bone Joint Surg [Am] 2003;85-A:259-65.

3. Pagnano MW, Cushner FD, Scott WN. Role of the posterior cruciate ligament in total knee arthroplasty. J Am Acad Orthop Surg 1998;6:176-87.

4. Gollehon DL, Torzilli PA, Warren RF. The role of the posterolateral and cruciate ligaments in the stability of the human knee: a biomechanical study. J Bone Joint Surg [Am] 1987;69-A:233-42.

5. Most E, Li G, Sultan PG, Park SE, Rubash HE. Kinematic analysis of conventional and high-flexion cruciate-retaining total knee arthroplasties: an in vitro investigation. J Arthroplasty 2005;20:529-35

6. Mihalko WM, Krackow KA. Posterior cruciate ligament effects on the flexion space in total knee arthroplasty. Clin Orthop 1999;360:243-50

7. Nakagawa S, Johal P, Pinskerova V, et al. The posterior cruciate ligament during flexion of the normal knee. J Bone Joint Surg [Br]2004;86-B:450-6.

8. Freeman MA, Pinskerova V. The movement of the knee studied by magnetic resonance imaging. Clin Orthop 2003;410:35-43.

9. Komistek RD, Dennis DA, Mahfouz M. In vivo fluoroscopic analysis of the normal human knee. Clin Orthop 2003;410:69-81.

10. Mahoney OM, Noble PC, Rhoads DD, Alexander JW, Tullos HS. Posterior cruciate function following total knee arthroplasty: a biomechanical study. J Arthroplasty 1994;9:569-78.

11. Matsuda S, Miura H, Nagamine R, et al. Knee stability in posterior cruciate ligament retaining total knee arthroplasty. Clin Orthop 1999;366:169-73.

12. Dejour D, Deschamps G, Garotta L, Dejour H. Laxity in posterior cruciate sparing and posterior stabilized total knee prostheses. Clin Orthop 1999;364:182-93.

13. Straw R, Kulkarni S, Attfield S, Wilton TJ. Posterior cruciate ligament at total knee replacement: essential, beneficial or a hindrance? J Bone Joint Surg $[\mathrm{Br}]$ 2003;85-B:671-4.

14. Victor J, Banks S, Bellemans J. Kinematics of posterior cruciate ligament-retaining and -substituting total knee arthroplasty: a prospective randomised outcome study. J Bone Joint Surg [Br] 2005;87-B:646-55.

15. Waslewski GL, Marson BM, Benjamin JB. Early, incapacitating instability of posterior cruciate ligament-retaining total knee arthroplasty. J Arthroplasty 1998;13:763-7.

16. Morberg $P$, Chapman-Sheath $P$, Morris $P$, Cain S, Walsh WR. The function of the posterior cruciate ligament in an anteroposterior-gliding rotating platform total knee arthroplasty. J Arthroplasty 2002;17:484-9.

17. Pagnano MW, Hanssen AD, Lewallen DG, Stuart MJ. Flexion instability after primary posterior cruciate retaining total knee arthroplasty. Clin Orthop 1998;356:39-46.

18. Yamakado K, Worland RL, Jessup DE, Diaz-Borjon E, Pinilla R. Tight posterior cruciate ligament in posterior cruciate-retaining total knee arthroplasty: a cause of posteromedial subluxation of the femur. J Arthroplasty 2003;18:570-4.
19. Swany MR, Scott RD. Posterior polyethylene wear in posterior cruciate ligamentretaining total knee arthroplasty: a case study. J Arthroplasty 1993;8:439-46.

20. Kim H, Pelker RR, Gibson DH, Irving JF, Lynch JK. Rollback in posterior cruciate ligament-retaining total knee arthroplasty: a radiographic analysis. J Arthroplasty 1997; 12:553-61.

21. Van Hal CT, Van Hellemond GG, Wymenga AB, Jacobs WCH. The anteriorposterior laxity after total knee arthroplasty inserted with a ligament tensor. Knee Surg Sports Traumatol Arthrosc 2007; in press.

22. Allain J, Goutallier D, Voisin MC. Macroscopic and histological assessments of the cruciate ligaments in arthrosis of the knee. Acta Orthop Scand 2001;72:266-9.

23. Nozaki H, Banks SA, Sugaro T, Hodge WA. Observations of femoral rollback in cruciate-retaining knee arthroplasty. Clin Orthop 2002;404:308-14.

24. Banks S, Bellemans J, Nozaki H, et al. Knee motions during maximum flexion in fixed and mobile-bearing arthroplasties. Clin Orthop 2003;410:131-8.

25. Li G, Zayontz S, Most E, et al. Cruciate-retaining and cruciate-substituting total knee arthroplasty: an in vitro comparison of the kinematics under muscle loads. $J$ Arthroplasty 2001;16:150-6.

26. Most E, Zayontz S, Li G, et al. Femoral rollback after cruciate-retaining and stabilizing total knee arthroplasty. Clin Orthop 2003;410:101-13.

27. Sorger JI, Federle D, Kirk PG, et al. The posterior cruciate ligament in total knee arthroplasty. J Arthroplasty 1997;12:869-79.

28. Misra AN, Hussain MR, Fiddian NJ, Newton G. The role of the posterior cruciate ligament in total knee replacement. J Bone Joint Surg [Br] 2003;85-B:389-92.

29. Archibeck MJ, Berger RA, Barden RM, et al. Posterior cruciate ligamentretaining total knee arthroplasty in patients with rheumatoid arthritis. J Bone Joint Surg [Am] 2001;83-A:1231-6.

30. Hartford JM, Banit D, Hall K, Kaufer H. Radiographic analysis of low contact stress meniscal bearing total knee replacements. J Bone Joint Surg [Am] 2001;83A:229-34.

31. Walker PS, Ambarek MS, Morris JR, Olanlokun K, Cobb A. Anterior-posterior stability in partially conforming condylar knee replacement. Clin Orthop 1995;310:87-97.

32. Dennis DA, Komistek RD, Mahfouz MR, Haas BD, Stiehl JB. Multicenter determination of in vivo kinematics after total knee arthroplasty. Clin Orthop 2003;416:37-57.

33. Bertin KC, Komistek RD, Dennis DA, et al. In vivo determination of posterior femoral rollback for subjects having a NexGen posterior cruciate-retaining total knee arthroplasty. J Arthroplasty 2002;17:1040-8.

34. Li G, Suggs J, Hanson G, et al. Three-dimensional tibiofemoral articular contact kinematics of a cruciate-retaining total knee arthroplasty. J Bone Joint Surg [Am] 2006;88-A:395-402. 